rung auf den Bias langzeitüberlebender Patienten - kein klarer Überlebensvorteil bei der neoadjuvanten Therapie gesehen (Hazard Ratio, HR =0,97). Die erste Aussage der Studie sei, dass wichtige Unter- schiede zwischen Patienten in klinischen Studien und der allgemeinen urologischen Praxis bestünden, sagte Hanna. Da nach der Adjustierung der Ergebnisse kein klarer Überlebensvorteil einer neo- adjuvanten Therapie für alle Patienten gezeigt werden konnte, solle die Identifizierung von Patienten, die von einer neoadjuvante Therapie profitieren, weiter vorangetrieben werden. Dr.Ine Schmale

\section{Active Surveillance auch beim nicht invasiven Harnblasenkarzinom?}

$D^{2}$ ie Active Surveillance (AS) könnte gemäß dem italienischen BIAS-Projekt auch für das nicht invasive Harnblasenkarzinom (NMIBC) eine Option darstellen [Hurle R, et al. EAU 2017, Poster \#655]. Das Harnblasenkarzinom mit guter Prognose (papilläres „low-grade“Karzinom, pTa) hat von allen Urothelialkarzinomen das beste Outcome, da Patienten zwar ein hohes Rezidiv-, jedoch nur ein sehr geringes Progressionsrisiko haben. An der prospektiven Längsschnittbeobachtungsstudie nahmen 99 Patienten mit "low-grade“ pTa- oder pTla-Tumoren in der Vorgeschichte teil, die nach transurethraler Resektion des
Blasentumors (TURBT) ein Rezidiv entwickelt hatten. Bei den Teilnehmern wurden im ersten Jahr alle vier und anschließend alle sechs Monate Urinzytologien und flexible Zystoskopien durchgeführt. Zwischen der ersten TURBT und dem Beginn der AS lagen im Median 22,5 Monate, die Zahl der vorherigen TURBT betrug im Median 2. Zu Beginn hatten 80 Patienten einen pTa- und 19 einen pTla-Tumor. Das Grading war zu diesem Zeitpunkt bei 69 Patienten G1 und bei 30 Patienten G2. Die Patienten nahmen im Median für 21,3 Monate an der AS teil. Bei 40 Patienten (40,4\%) trat ein Versagen ein, definiert als jegliche
Progression, das heißt Zunahme von Zahl oder Durchmesser der Läsionen, positive Zytologie, persistierende Makrohämaturie oder Notwendigkeit jeder weiteren Intervention. Bei keinem Patienten verschlechterten sich das Stadium und das Grading. Bei elf Patienten mit AS-Versagen lag ein pT1a- und bei 31 ein pTa-Tumor vor; sechs AS-Versager wurden als G3, neun als G2 und 27 als G1 klassifiziert. Die Adhärenz der Patienten gegenüber den AS-Untersuchungen lag bei $95 \%$. Die Autoren kommen zu dem Schluss, ihre Studie würde frühere Ergebnisse in einer größeren Kohorte bestätigen, wonach die AS eine vertretbare Option für Patienten mit rezidiviertem, kleinem, „low-grade“, papillärem Harnblasenkarzinom in frühem Stadium nach TURBT sein könnte. Petra Eiden

\title{
Neue Therapiewege beim Nierenzellkarzinom
}

$D^{\text {ie }}$ ie einzige kurative Therapie beim Nierenzellkarzinom ist die partielle oder radikale Nephrektomie. Patienten mit Nierenzellkarzinom, für die Nephrektomie oder ablative Verfahren nicht geeignet sind, benötigen neue Therapiestrategien. Peter Aslan, Sydney/Australien, stellte Ergebnisse der ersten Studie zur SIRT („selective internal radiation therapy") bei Nierenzellkarzinompatienten vor [Aslan P, et al. EAU 2017, Poster \#360]. Bei der SIRT werden radioaktive Kügelchen, sogenannte Mikrosphären, über eine Nierenarterie direkt in das gut durchblutete Tumorgewebe appliziert. Das in den Mikrosphären enthaltene Yttrium-90 strahlt mit hoher lokaler Wirkung über mehrere Tage hinweg in das Tumorgewebe. In der RESIRT-Studie erhielten insgesamt 21 Patienten eine SIRT in verschiedenen Dosierungen. Nach der SIRT wurden die Patienten über zwölf Monate in regelmäßigen Abständen kon- trolliert. Primäre Endpunkte der Studie waren die Sicherheit und die Toxizität 30 Tage nach Applikation. Die sekundären Studienendpunkte enthielten die Sicherheit und Toxizität zu anderen Zeitpunkten, die renale Funktion und das Tumoransprechen. Zum Zeitpunkt des Studieneinschlusses waren die Patienten durchschnittlich 75 Jahre alt, nahezu ein Viertel war im $\mathrm{CKD}$ (,,chronic kidney disease")-Stadium 1, ein Fünftel im Stadium 2 und mehr als die Hälfte der Patienten im CKD-Stadium 3. Die mediane Nachbeobachtungszeit bei Auswertung der präsentierten Daten betrug 12,0 Monate. 14 Patienten hatten die zwölf Monate Nachbeobachtungszeit komplettiert, fünf Patienten verstarben vor Studienende, ein Patient zog nach neun Monaten Nachbeobachtungszeit seine Einwilligung zurück und ein Patient war bei Auswertung noch in der Studie. Während der ersten 30 Tage nach der SIRT berichteten $85,7 \%$ der Patienten über Nebenwirkungen. Die der SIRT zugeordneten Nebenwirkungen waren alle von Grad 1-2 und nicht klinisch relevant. Während der zwölfmonatigen Nachbeobachtungszeit wurde bei allen 21 Patienten von Nebenwirkungen berichtet, bei jeweils fünf Patienten von wenigstens Grad 3 sowie Grad 5. Keine der schweren Nebenwirkungen oder der Grad $\geq 3$-Ereignisse wurde mit der SIRT assoziiert. Auch in Bezug auf die renale Funktion erwies sich die SIRT als sicher, sogar bei Patienten mit grenzwertiger Nierenfunktion. Das beste renale Tumoransprechen war eine partielle Remission bei $2 / 20 \mathrm{~Pa}-$ tienten $(10 \%)$ sowie eine stabile Erkrankung bei 17/20 Patienten (85\%) und ein Pseudoprogress, der sich später als stabile Erkrankung erwies. In dieser Pilotstudie habe sich die SIRT mit Y-90-Mikrosphären in allen Dosierungen als machbare Therapieoption für Nierenzellkarzinompatienten gezeigt, die für eine konventionelle Therapie nicht geeignet sind, so das Fazit der Autoren. Dr. Ine Schmale 